\title{
Human Dendritic Cells in the Severe Combined Immunodeficiency Mouse Model: Their Potentiating Role in the Allergic Reaction
}

\author{
Hamida Hammad, Catherine Duez, Olivier Fahy, Anne Tsicopoulos, Claude André, \\ Benoît Wallaert, Serge Lebecque, André-Bernard Tonnel, and Joël Pestel \\ Unité INSERM U-416 (HH, CD, OF, AT, BW, A-BT, JP), Institut Pasteur de Lille; Département de Pneumologie (AT, \\ BW, A-BT), Hôpital A. Calmette, CHRU de Lille, Lille Cedex; Stallergenes S.A. (CA), Fresnes; and Schering Plough \\ (SL), Dardilly, France
}

\begin{abstract}
SUMMARY: Dendritic cells (DCs) are present in the lungs and airways of healthy and allergic subjects where they are exposed to inhaled antigens. After the uptake of antigens, DCs migrate to lymphoid organs where T cells initiate and control the immune response. The migratory properties of DCs are an essential component of their function but remain unclear in the situation of allergic diseases. To better understand the role of DCs in response to allergens, we first investigated their presence in an original experimental model of allergic asthma: the humanized severe combined immunodeficiency (SCID) mouse reconstituted with peripheral blood mononuclear cells from patients sensitive to Dermatophagoides pteronyssinus (Dpt). Human DCs were detected in lungs of mice developing an inflammatory pulmonary infiltrate and appeared to be mainly located in the alveolar spaces. In a second step, human DCs were generated in vitro from monocytes and injected into naive SCID mice exposed or not exposed to Dpt aerosols. Their migratory behavior was explored, as well as their potential role in modulating the IgE production after exposure to Dpt. After exposure to Dpt, the number of DCs present in airways decreased, while it increased into the spleen and thymus of the mice. The IgE production increased in the presence of DCs as compared with mice not injected with DCs. These results suggest that DCs may play a role in the pulmonary allergic reaction developed in response to Dpt in SCID mice. (Lab Invest 2000, 80:605-614).
\end{abstract}

A Ilergic asthma is an inflammatory disease characterized by three main features. The first characteristic is an allergen-induced IgE antibody production that depends on direct interactions between CD40, expressed by B cells, and its ligand CD40L, expressed by $T$ cells. After this initial interaction, $T$ cells produce IL-4 and IL-13, two Th2 cytokines involved in the immunoglobulin class switch toward IgE (Abbas et al, 1996). The second feature is an inflammatory reaction characterized by a cellular infiltrate composed of eosinophils and lymphocytes. The third feature is the development of an airway hyperresponsiveness (AHR). The three characteristic features of allergic asthma are present in the humanized severe combined immunodeficiency (SCID) mice model. After intraperitoneal reconstitution with mononuclear cells and exposure to Dermatophagoides pteronyssinus (Dpt) aerosols, SCID mice produced human IgE antibodies (Pestel et al, 1994) and developed a pulmonary inflammatory reaction mainly characterized by an infiltrate of activated $\mathrm{CD}^{+}{ }^{+} \mathrm{CD}_{45 \mathrm{RO}^{+}} \mathrm{HLA}^{-D R^{+}} \mathrm{T}$ cells (Duez et al, 1996; 1998). Moreover, allergic hu-SCID

Received December 30, 1999.

Address reprint requests to: Dr. J. Pestel, INSERM U416, Institut Pasteur de Lille, 1 rue du Professeur Calmette, B.P 245, 59019 Lille cedex, France. Fax: 333208773 45; E-mail: Joel.Pestel@pasteur-lille.fr mice exhibited an airway hyper-responsiveness to carbachol (Duez et al, 2000).

Because the allergic reaction is mainly mediated by $\mathrm{T}$ cells, the role of dendritic cells (DCs), one of the most potent antigen-presenting cells, in the development of allergic reactions was investigated. Some evidence suggests a potential role of DCs in allergy. First, dendritic cells are located in human lungs, particularly in the epithelium and in the alveolar spaces and walls. Their number increases in the lungs of atopic patients (Bellini et al, 1993; Moller et al, 1996) where they form a network involved in the uptake of inhaled antigens. Second, DCs express the high affinity $\lg$ E receptor, which is implicated in the capture and the presentation of allergens to $\mathrm{T}$ cells (Semper et al, 1995). In addition, in vitro experiments suggest that human DCs can interact with CD45RA ${ }^{+}$T cells, which are involved in the initiation of IgE production (Patel et al, 1996). In the mouse the selective depletion of airway DCs was demonstrated to lead to a dramatic reduction in the number of $B$ and $T$ lymphocytes in bronchoalveolar lavages and in Type 2 cytokine-associated eosinophilic airway inflammation (Lambrecht et al, 1998). However, approaches to evaluate the potential role of human DCs in the allergic reaction are limited because of ethical constraints. Indeed, because SCID mice could be used to further analyze the behavior of human DCs in a more physi- 
ological environment, this model was used to better investigate the role of DCs in the allergic reaction.

In this study, the involvement of DCs in the development of the allergic reaction after a challenge with common aeroallergens, such as the house dust mite Dpt, in humanized SCID mice was investigated. Consequently, we first studied the presence of dendritic cells in different organs of SCID mice reconstituted with peripheral blood mononuclear cells (PBMC) from allergic patients. In a second step, the migratory behavior of dendritic cells generated in vitro from human monocytes was explored in naive SCID mice exposed or not exposed to Dpt aerosols by analyzing three different organs: the lung, spleen, and thymus. Moreover, the in vitro effect of Der $p 1$, the major allergen of Dermatophagoides pteronyssinus, on the biological activity of monocyte-derived DCs was explored. The modulatory effect of Der $\mathrm{p} 1$ on the expression of the adhesion molecule CD11c (highly expressed by DCs and involved in their migration from nonlymphoid to lymphoid organs) was investigated, as well as the impact of Der $\mathrm{p} 1$ on the production of $\mathrm{TNF} \alpha$, a pro-inflammatory and dendritic cell maturing cytokine. In the last part of this study, the capacity of DCs to modulate an IgE versus the IgG production was analyzed in SCID mice reconstituted with peripheral blood mononuclear cells (PBMC) from allergic patients and injected or not injected with autologous DCs.

\section{Results}

\section{Detection of Human Dendritic Cells in PBMC hu-SCID Mice by Immunohistochemistry}

As previously described in other works (Duez et al, 1996; 1998), the number of human CD45 ${ }^{+}$cells in the lungs of SCID mice reconstituted with PBMC from allergic patients was largely higher $\left(90 \pm 7\right.$ cells $\left./ \mathrm{mm}^{2}\right)$ than the number of human cells detected in the lungs of SCID mice reconstituted with PBMC from healthy donors $\left(6 \pm 1\right.$ cells $\left./ \mathrm{mm}^{2}\right)$. Interestingly, when the mice were reconstituted with $\mathrm{PBMC}$ from asthmatic allergic patients, the number of $\mathrm{CD} \mathrm{a}^{+}$cells was 8 -fold higher than in nonallergic SCID mice $(p=0.02)$. Moreover, the number of mature DC-LAMP ${ }^{+}$cells was 10-fold higher in the lungs of allergic PBMC hu-SCID mice compared with the controls (Fig. 1). DCs from patients with asthma were mainly located in the alveoli. Both mature (DC-LAMP+) and immature (DCGM4+) DCs were detected in the lungs of allergic PBMC hu-SCID mice. In the broncho-alveolar lavage fluids of allergic PBMC hu-SCID mice, mature DC (CD83+, DC$\mathrm{LAMP}+$ ) represented $0.8 \%$ and immature DC (CD1a+, DCGM4+) only $0.2 \%$ of the total cell number.

In the spleen, only a few DCs $\left(1 / \mathrm{mm}^{2}\right)$ were detected when the mice were reconstituted with PBMC from either allergic patients or healthy donors. In the thymus, no DCs could be detected in SCID mice reconstituted either with PBMC from allergic patients or with PBMC from nonallergic donors (data not shown).

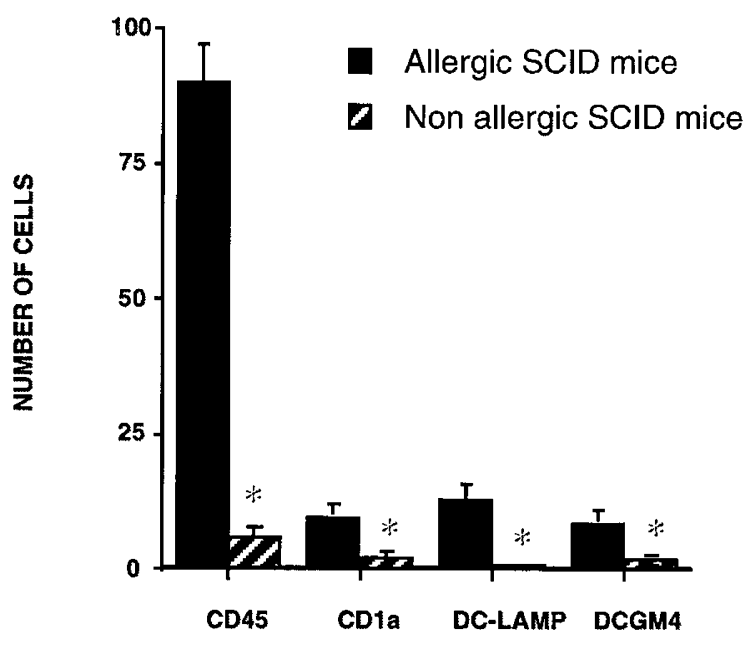

Figure 1.

Number of human cells expressing CD1a, DC-LAMP, or DCGM4 in the lungs of SCID mice reconstituted with peripheral blood mononuclear cells (PBMC) from allergic patients versus healthy donors. Results are expressed as mean \pm SEM per $\mathrm{mm}^{2} .{ }^{*} p$ values $\leq 0.05$.

\section{Phenotype of Dendritic Cells Differentiated In Vitro from Monocytes}

Dendritic cells detected in the lungs of PBMC hu-SCID mice suggest that PBMCs are able to differentiate into DCs after their injection into hu-SCID mice. Because it has been previously shown that DCs can be generated in vitro from PBMCs in the presence of GM-CSF and IL-4, we have compared the phenotype of DC generated from PBMC isolated from both healthy donors $(n=8)$ and allergic patients $(n=10)$. After 14 days of culture in presence of GM-CSF and IL-4, flow cytometry analysis revealed the presence of two cell populations. The first one displayed the same size as monocytes but expressed low levels of CD14 and CD1a markers. The second population was constituted of large cells expressing low levels of CD14 marker but high levels of CD1a marker. Dendritic cells from healthy subjects expressed high levels of CD1a $(\mathrm{MFI}=1307.4 \pm 280.5)$ and low levels of CD83, a marker of mature DCs (MFI $=8.02 \pm 3.7)$. They also expressed the costimulatory molecules CD80 (MFI = $75.49 \pm 26.3)$ and CD86 (MFI = $75.72 \pm 23.95)$. One representative experiment is shown in Figure 2.

Dendritic cells from allergic patients also expressed costimulatory molecules CD80 (MFI = $46.89 \pm 7.31)$ and CD86 (MFI = $123.3 \pm 20.21$ ) (Fig. 2). Interestingly, dendritic cells from atopic patients presented a totally different surface phenotype than cells from healthy patients; they expressed low levels of CD1a (MFI = $144.97 \pm 29.19)$ and high levels of CD83 (142.04 \pm 7.26) on their membrane compared with DCs from healthy donors (Fig. 3).

\section{Detection of Human Dendritic Cells in DC hu-SCID Mice by Immunohistochemistry}

In this part of the experimental protocol, SCID mice were exclusively reconstituted with $1 \times 10^{6}$ purified 


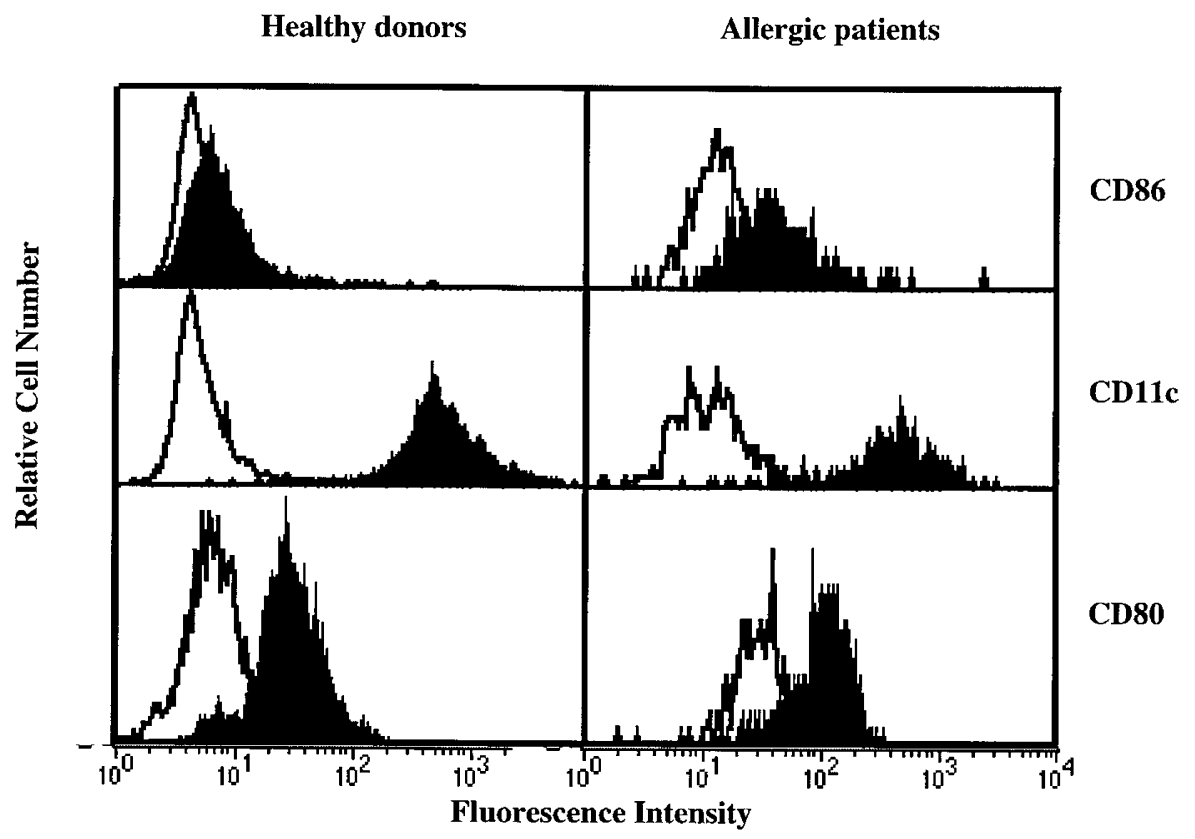

Figure 2.

CD86, CD11c, and CD80 expression by monocyte-derived Dendritic cells (DCs) from healthy donors (left) and from allergic patients (right). Monocytes from healthy donors and from allergic patients were cultured for 14 days in the presence of GM-CSF and IL-4. The cells were incubated with PE-labeled anti-CD86 and anti-CD11C or with FITC-labeled anti-CD80. Monocyte-derived DCs were electronically gated and analyzed for CD86 (filled histograms, top), CD11c (filled histograms, middle), and CD80 (filled histograms, bottom) expression. Open histograms, reactivities of fluorochrome-matched isotype.

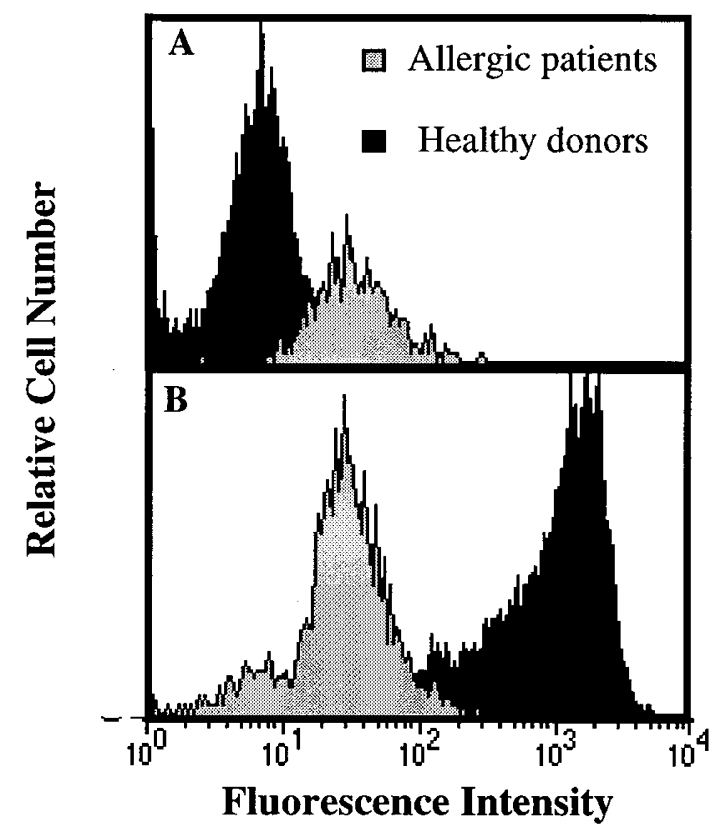

Figure 3.

Comparison of the stage of maturation between DCs from healthy donors and from allergic patients. Monocyte-derived DCs from allergic patients expressed the marker of mature cells CD83 (A) more than DCs from healthy donors. In contrast, DCs from allergic patients expressed the marker of immature cells CD1a (B) less than DCs from healthy donors.

human dendritic cells but without previous engraftment with PBMC. The only experimental variable was represented by the fact that mice were exposed or not exposed to the allergen. In mice injected with purified DCs from healthy donors without consecutive expo- sure to the allergen aerosol, dendritic cells were detectable in the lung $\left(3 \pm 0.7 \mathrm{CD} \mathrm{a}^{+} \mathrm{DCs} / \mathrm{mm}^{2}\right)$ but also in the spleen $\left(10 \pm 0.7 \mathrm{CD} \mathrm{a}^{+} \mathrm{DCs} / \mathrm{mm}^{2}\right)$ and in the thymus $\left(2 \pm 0.4 \mathrm{CD} \mathrm{a}^{+} \mathrm{DCs} / \mathrm{mm}^{2}\right)$ of the mice.

When the mice were exposed to an aerosol of Dpt, CD1a + cells were also detected in the three collected organs, however the number of cells detected in the lungs was lower than in mice not exposed to Dpt $(2 \pm$ $0.9 \mathrm{DC} / \mathrm{mm}^{2}$ ). In contrast, the number of CD1a+ cells detected in the spleen and in the thymus of the animals was higher after allergen exposure $(15 \pm 1.1$, $p=0.005$ and $4 \pm 0.4 \mathrm{DCs} / \mathrm{mm}^{2}, p=0.01$, respectively, as compared with mice not exposed to Dpt) (Fig. 4A). When DCs were obtained from blood samples of allergic asthmatic patients, the number of CD1a+ cells detected in the lungs, spleen, and thymus of the mice was higher (respectively $8 \pm 0.7,16 \pm$ 0.4 and $4 \pm 0.7 \mathrm{DCs} / \mathrm{mm}^{2}$ ) by comparison with DCs from healthy donors ( $p<0.05)$ (Fig. 4B). When the mice were exposed to Dpt, the cell number decreased in the lungs $\left(2 \pm 1.1 \mathrm{DCs} / \mathrm{mm}^{2}, p=0.001\right)$ as compared with mice not exposed to Dpt but increased in the lymphoid organs, namely the spleen and the thymus $\left(24 \pm 0.4 \mathrm{DCs} / \mathrm{mm}^{2}\right.$ in the spleen $p=0.01$ and $6 \pm 0.9 p=0.18 \mathrm{DCs} / \mathrm{mm}^{2}$ respectively) as compared with mice not exposed to Dpt.

\section{Detection of Dpt-Pulsed DC in Lymphoid Organs of DC hu-SCID Mice}

To better understand why the number of human DCs was increased in the lymphoid organs after allergen exposure, the detection of allergen-positive DCs was investigated in different lymphoid organs (the spleen 

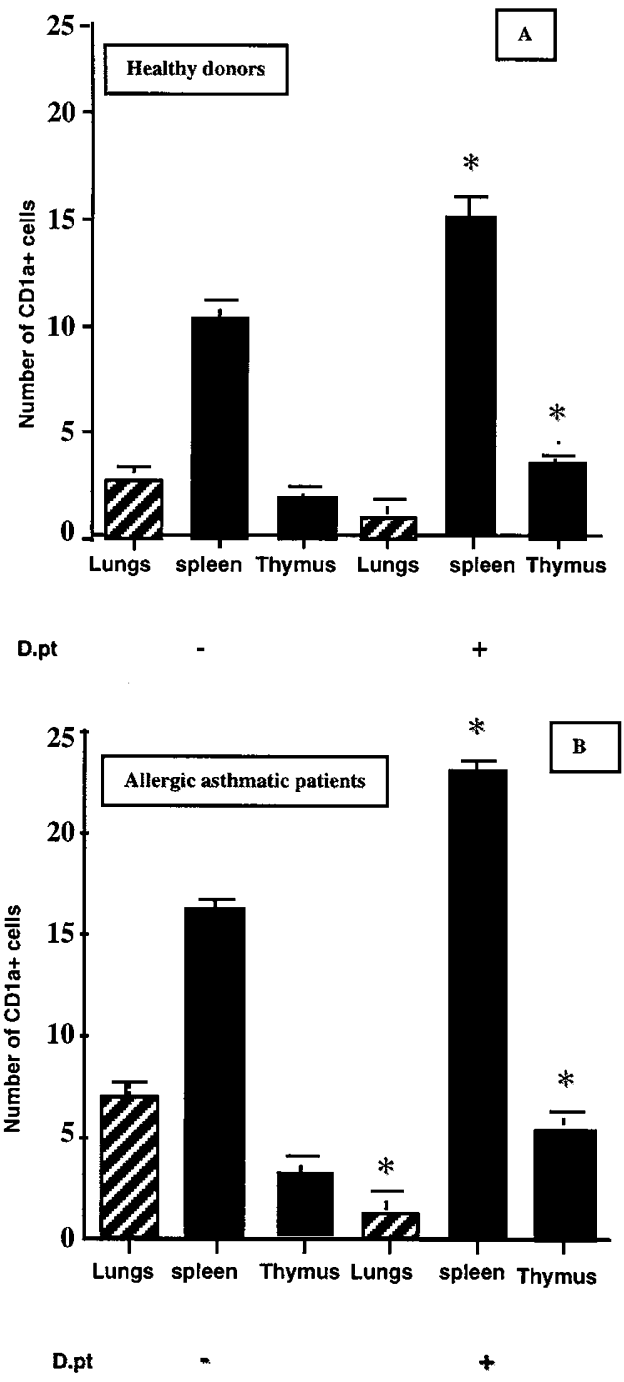

Figure 4.

Number of CD1a+ dendritic cells detected in the lungs, spleen, and thymus of SCID mice exposed or not exposed to allergen aerosol, 7 days after mice reconstitution with DCs from healthy donors (A) or from allergic patients (B). Results are expressed as the mean number of cells \pm SEM per $\mathrm{mm}^{2} .{ }^{*} p$ values $\leq 0.05$.

and the inguinal lymph nodes) of the mice before and after Dpt inhalation by using a specific anti-Der $p 1$ rabbit serum. As shown in Figure $5 \mathrm{~A}$, before allergen exposure, in the lymphoid organs of DC hu-SCID mice $(n=6)$ reconstituted with DCs from healthy donors, no allergen-loaded DCs were detected. In contrast, after Dpt inhalation, allergen-loaded DCs were found in the spleen $\left(8 \pm 2\right.$ cells $\left./ \mathrm{mm}^{2}\right)$ and the lymph nodes $(7 \pm 2$ cells $/ \mathrm{mm}^{2}$ ) of these mice $(n=7)$. Similar effects were observed in mice $(n=12)$ reconstituted with DCs from allergic patients (Fig. 5B). In contrast when spleen sections of SCID mice reconstituted with DCs from healthy donors or from allergic patients and exposed to Dpt allergen were analyzed with a serum from nonimmunized rabbits or with a serum from rabbits immunized with a nonimmunogenic Der $\mathrm{p} 1$ peptide, no positive cells were detected.
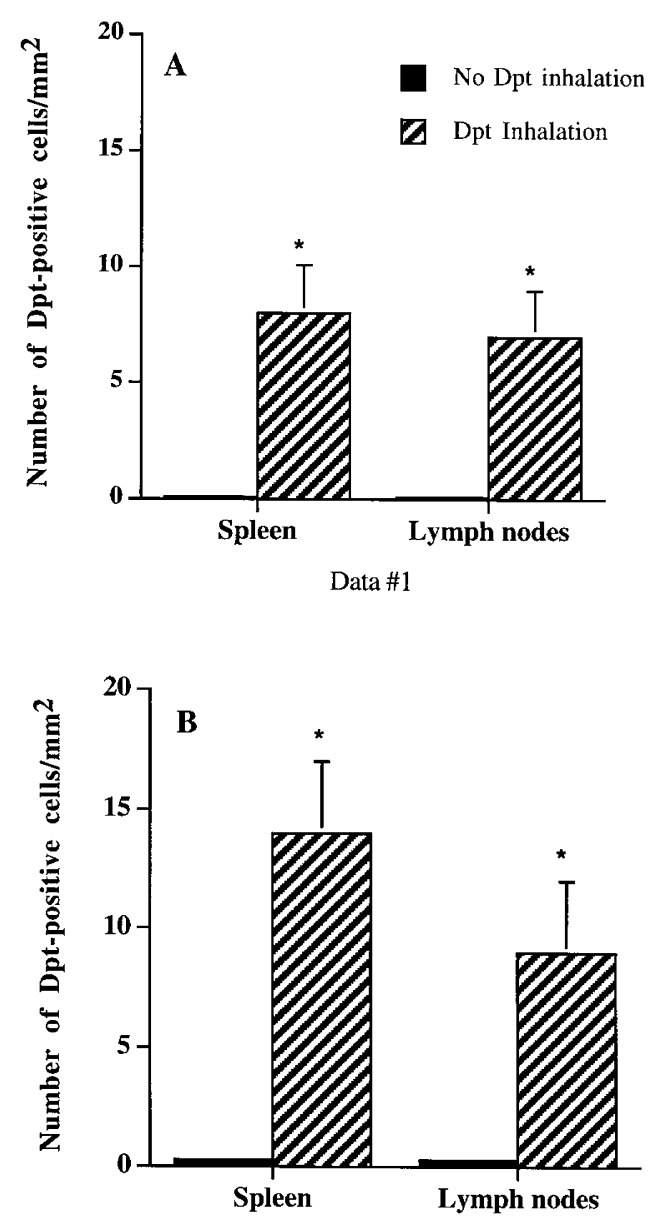

Figure 5.

Number of Dpt-positive dendritic cells detected in the lymphoid organs (spleen and lymph nodes) of SCID mice reconstituted with DCs from healthy donors (A) or allergic patients (B) and exposed or not exposed to allergen aerosol. Results are expressed as the mean number of cells \pm SEM per $\mathrm{mm}^{2}$. ${ }^{*} p$ values $\leq 0.05$.

\section{Allergen-Induced Modulation of CD11c Expression}

Monocyte-derived DCs from both healthy donors $(n=$ $5)$ and allergic patients $(n=8)$ that were cultured for 14 days with GM-CSF and IL-4 highly expressed CD11C on their membrane (MFI $=663.73 \pm 66.94)$. After 7 days of incubation with Der $p 1$, the major allergen of the house dust mite Dpt, CD11c was found to be up-regulated on DCs (MFI: $1087.15 \pm 157.41$ ) (Fig. 6).

\section{TNFa Production by Monocyte-Derived DCs}

TNF $\alpha$ production was analyzed after exposure of DCs to $100 \mathrm{ng} / \mathrm{ml}$ Der $\mathrm{p} 1$ or to $100 \mathrm{ng} / \mathrm{ml}$ tetanus toxin for 24, 48, and 72 hours. At baseline, DCs not exposed to Der $\mathrm{p} 1$ were shown to produce TNF $\alpha$. However, after exposure to Der $\mathrm{p} 1$ as shown in Figure 7, DCs from allergic patients (Fig. 7A) and from healthy donors (Fig. 7B) significantly increased TNF $\alpha$ production ( $p \leq$ 0.05). Interestingly, after 72 hours, only DCs from allergic patients continued to produce high amounts of TNF $\alpha$. Moreover, DCs from allergic patients (Fig. 7A) and from healthy donors (Fig. 7B) exposed to the 


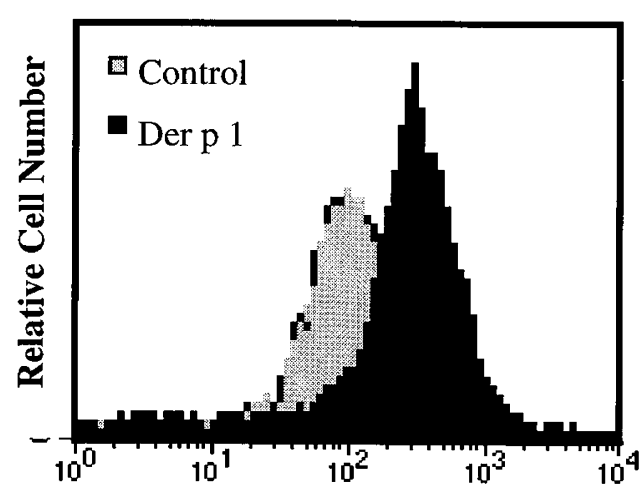

Fluorescence Intensity

\section{Figure 6.}

CD11c expression by monocyte-derived DCs. DCs cultured for 7 days in the presence of Der $p 1$, the major allergen of Dpt, up-regulate their expression of CD11c.
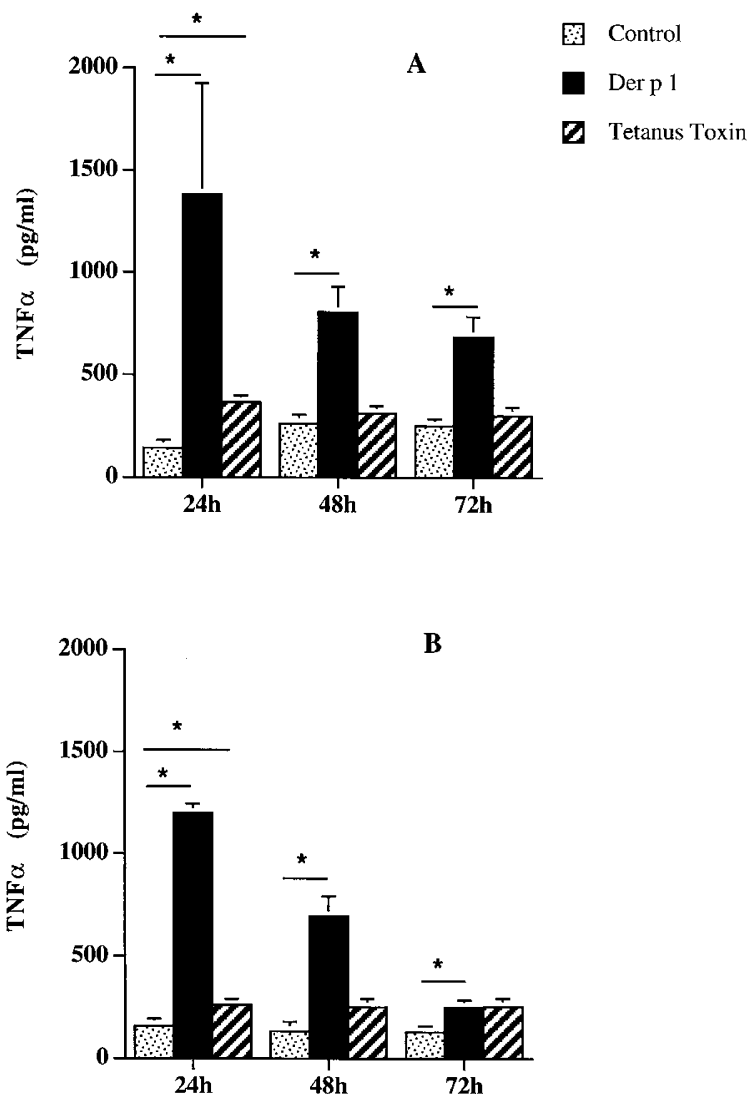

\section{Figure 7.}

Exacerbation of TNF $\alpha$ production by dendritic cells from allergic patients (A) and from healthy donors (B) after 24,48 , and 72 hours exposure to $100 \mathrm{ng} / \mathrm{ml}$ Der $p 1$ or to $100 \mathrm{ng} / \mathrm{ml}$ tetanus toxin. The control corresponds to dendritic cells cultured with GM-CSF and IL-4. Data are shown as mean \pm SEM. ${ }^{*} p$ values $\leq 0.05$.

tetanus toxin significantly increased their TNF $\alpha$ production after 24 hours. However, the toxin-induced amounts of $\mathrm{TNF} \alpha$ were lower than the allergeninduced amounts. After 48 and 72 hours, the amount of $\mathrm{TNF} \alpha$ released by the DCs in response to the tetanus toxin were not significantly different from those produced by unstimulated DCs. In conclusion, Der $\mathrm{p} 1$ seemed to favor a sustained TNF $\alpha$ production.

\section{Modulation of the IgE/lgG Production by Dendritic Cells}

In the sera of mice $(n=13)$ reconstituted only with PBMC from healthy donors $(n=4)$, low concentrations of human $\operatorname{lgE}(1.6 \pm 0.99 \mathrm{IU} / \mathrm{ml})$ were detected at Week 1. IgE production did not change at Weeks 2 and 3 . When the mice $(n=13)$ were previously reconstituted with $\mathrm{DCs}$, the level of IgE detected in the murine sera increased $(10.3 \pm 4.7 \mathrm{IU} / \mathrm{ml})$ as soon as at Week 1, but the variations appeared to be nonsignificant up to Week 3 (Fig. 8A). However, in the presence of DCs, the IgG production was significantly increased as compared with mice injected with PBMC only (Fig. 8B).

In mice $(n=13)$ reconstituted only with PBMC from allergic patients with low levels of $\lg E(n=4)$, the concentrations of human IgE were higher $(6.7 \pm 2.2$ $\mathrm{IU} / \mathrm{ml} ; p<0.05)$ than those found in mice reconstituted with PBMC of healthy donors at Week 1. IgE production remained constant at Week $2(7.7 \pm 2.5$ $\mathrm{IU} / \mathrm{ml})$ and decreased at Week 3 . In mice $(n=12)$ reconstituted previously with DCs of allergic patients, the $\lg E$ production significantly increased at Week 1 $(43 \pm 22.3 \mathrm{lU} / \mathrm{ml} ; p<0.05)$ as compared with mice reconstituted only with $\mathrm{PBMC}$ and remained at a high level up to Week 3 (Fig. $8 \mathrm{C}$ ). Moreover, in mice injected with cells from allergic patients, the presence of DCs did not have any effect on the IgG production as compared with mice injected with PBMC only (Fig. 8D).

\section{Discussion}

The purpose of this work was to investigate the role of DCs in the allergic reaction. In a first step, the presence of dendritic cells in the lungs of SCID mice previously reconstituted with PBMC of atopic patients or healthy donors was investigated. When exposed to allergen aerosols, humanized SCID mice produced $\lg \mathrm{E}$ and developed a pulmonary inflammatory infiltrate consisting of human CD45+ cells, primarily CD4+ T cells (Duez et al, 1996). The present results show that human dendritic cells are detectable in lungs of huSCID mice developing a lung infiltrate. These cells were mainly located in the alveoli. In humans, previous works have already shown the presence of DCs at a similar place, however despite their low frequency ( $0.4 \%$ of the cells collected in bronchoalveolar lavage), they were closely associated to the initiation of the immune response in airways (Van Haarst et al, 1994). The DCs found in the lungs of hu-SCID mice were mainly mature cells. This maturation may be due to the allergen itself. It is known that after contact with an antigen, DCs become mature and increase the CD83 expression. The mechanism of DC maturation is still not completely understood. However, it is known that TNF $\alpha$ acts as an important maturation factor (Lore et al, 1998), and we demonstrated here that DCs from healthy donors and from allergic patients exposed in 

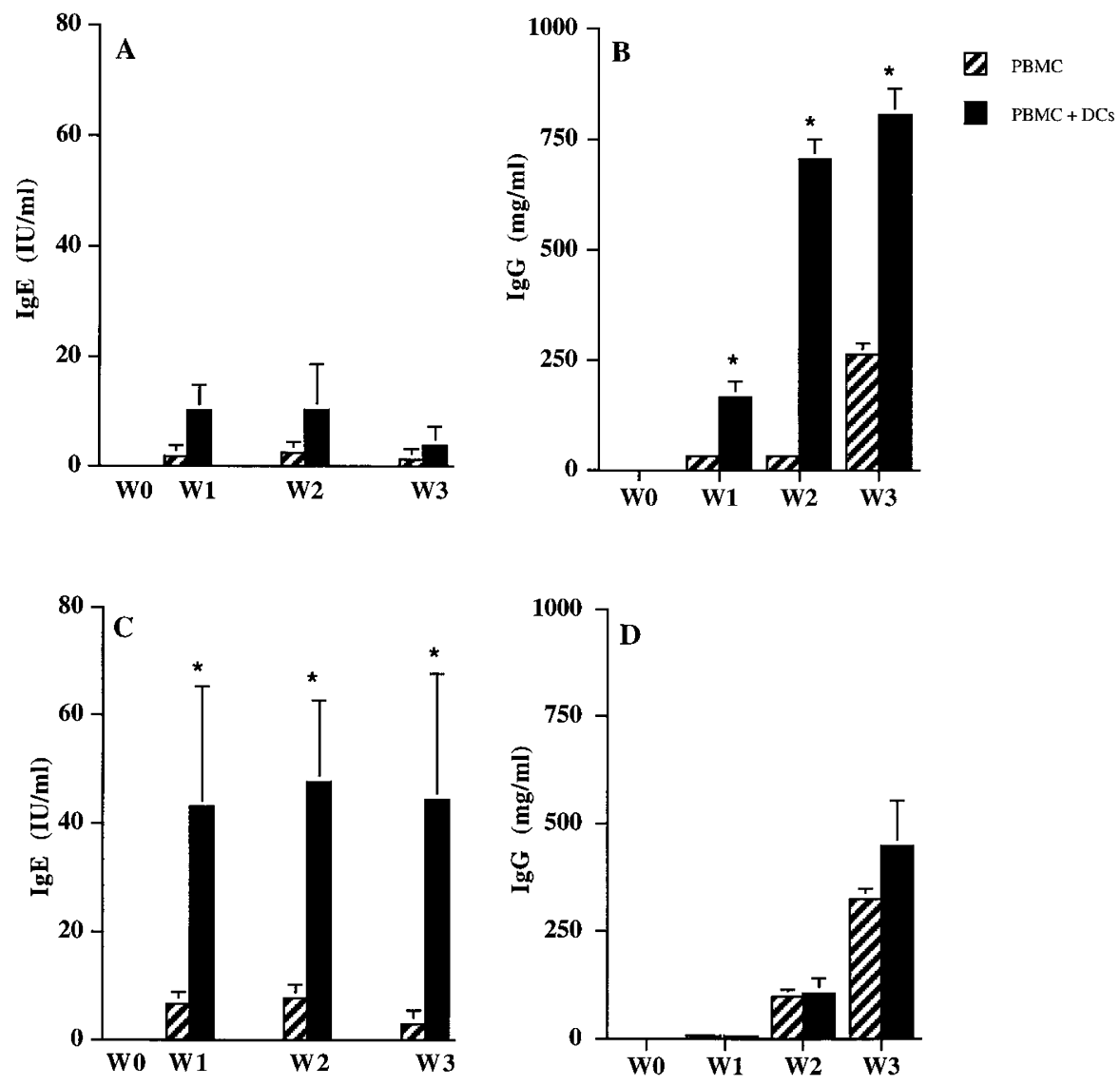

Figure 8.

Human IgE production in mice injected with PBMC or PBMC and DCs from healthy donors (A) or allergic asthmatic patients (C). The human IgG production was also measured in the sera of mice reconstituted with PBMC or PBMC and DCs from healthy donors (B) or allergic asthmatic patients (D). Data are shown as mean \pm SEM. ${ }^{*} p$ values $\leq 0.05$

vitro to Der p 1, a major allergen of Dermatophagoides pteronyssinus, lead to a rapid increase in TNF $\alpha$ production. In the case of DC from allergic patients, this increase of TNF $\alpha$ production lasted longer than in DC from healthy donors, certainly explaining the higher number of mature DCs encountered in allergic PBMC hu-SCID mice.

To better understand the behavior of DCs in allergic disease, we have studied their migration after a single injection in naive SCID mice of human DCs derived from monocytes cultured for 14 days with GM-CSF and IL-4. We analyzed the migratory behavior of DCs. The cells that we obtained in culture expressed different DC surface markers, such as CD1a or CD83. The expression of CD1a in our experience was heterogeneous and varied from one donor to the other. Nevertheless, more than $70 \%$ of cells derived from monocytes of nonallergic patients expressed CD1a. The second population of cells expressed low levels of CD14 and might represent precursors of DCs. During the differentiation into DCs, monocytes lose the expression of CD14 and acquire the CD1a marker (Cella et al, 1997). In this study, we have shown that dendritic cells from atopic patients expressed low levels of CD1a and expressed the mature dendritic cell marker CD83 compared with cells from nonatopic patients.
These data suggest that cells obtained by the culture of monocytes from nonatopic patients were immature cells. In contrast, dendritic cells from atopic patients were mature. Generally, culture medium supplemented with GM-CSF and IL-4 is used to obtain immature cells. To obtain mature dendritic cells, other cytokines such as IL-1 $\beta$ and TNF $\alpha$ or prostaglandin E2 have to be added in the culture medium (Kalinski et al, 1998). In our study, no TNF $\alpha$ or IL-1 $\beta$ was added. Therefore, one of the ways for cells from atopic patients to mature might be to produce one of these cytokines, such as TNF $\alpha$ (Lore et al, 1998), acting in an autocrine manner. Moreover, it is known that mature DCs are more efficient in the stimulation of naive $T$ cells (Palucka et al, 1998).

In this study, we showed that human dendritic cells injected intraperitoneally into naive SCID mice were capable of migrating even in the absence of an exogenous stimulation (allergen aerosol) and were located in different murine organs. The way of administration of the cells is critical in this kind of study. It has been shown in monkeys that when dendritic cells were injected intravenously, they were located mainly in the spleen, where they were eliminated. In contrast, in the same monkey model after subcutaneous injection, dendritic cells derived from monocytes cultured with 
GM-CSF and IL-4 could migrate without any stimulation and were located in draining lymph nodes 5 days later (Barratt-Boyes et al, 1997).

In the SCID model, dendritic cells injected in the peritoneal cavity were able to migrate to the lungs, where they were detected 7 days after the reconstitution of the mice. The cell number detected remained very low. This migration occurred without any stimulation. A kinetic of DC migration was performed. The lungs, spleen, and thymus of the mice were collected 1,3 , and 5 days after the cell reconstitution. The results showed that DCs were detected in the lungs of the mice 1 day after the cell transfer, suggesting that DCs have high capacities of migration.

After allergen exposure, the number of cells detected in the mouse lungs decreased. The presence of dendritic cells in the lungs suggests that these cells may be involved in the inhaled antigen uptake. Their decreasing number after allergen inhalation can be explained by the fact that the cells located in the lungs before the inhalation took up the allergen, left the lungs and migrated to lymph nodes or lymphoid organs, where they might induce a $\mathrm{T}$ cell dependent immune response. Recent studies showed that after Toluene Diisocyanate (TDI) inhalation in a guinea pig model, the number of dendritic cells increased in lungs approximately 24 hours after exposure to the hapten. A few hours later, the number of DCs decreased in the airways (Ban et al, 1997). In DC hu-SCID mice, dendritic cells were also located in lymphoid organs of the mice: the spleen and the thymus of the animals. The number of cells detected was higher in mice exposed to the house-dust mite aerosol. In our study, allergenpositive DCs from healthy donors were located, after inhalation, in the spleen and peripheral lymph nodes. In our experimental conditions, the potential reactivity against the components of CFA adjuvant was partially excluded by using a serum from rabbit immunized with a nonimmunogenic peptide of Der $p 1$ but in the presence of CFA. As in SCID mice reconstituted with DCs from healthy donors without inhalation, no allergen-positive DCs were detected in the lymphoid organs, these results suggest that nonallergen-loaded DCs can migrate from the peritoneal cavity to the lungs where, exposed to the allergen, they take it up and leave the lungs, heading toward the spleen and lymph nodes. Thus, these results suggest that GMCSF and IL-4-driven DCs introduced by the peritoneal route into SCID mice can migrate to the lungs and acquire the same behavior as lung DCs.

In the spleen, dendritic cells may initiate the response of $\mathrm{CD}^{+}$and $\mathrm{CD}^{+}{ }^{+} \mathrm{T}$ cells (Leenen et al, 1998). Moreover, DCs may contribute to the initiation of the production of IgE in the spleen, where germline epsilon immunoglobulin heavy chain transcripts are expressed by B cells (Goodman and Gerondakis, 1997). The reason why dendritic cells migrate from the peritoneal cavity to the lungs of SCID mice is still unclear. Dendritic cells are sentinels of the immune system, capable of circulating from one organ to the other. This migration could depend on the adhesion molecule network. Several studies have shown that dendritic cells express membrane adhesion molecules, such as $\beta 2$ integrins CD11a/CD18 and CD11c/ CD18 (McCarthy et al, 1997). Indeed, the migratory capacities of human dendritic cells in mice could depend on interactions between human $\beta 2$ integrins and murine ICAM1 expressed by endothelial cells. The murine molecule called MALA-2 displays high homologies with the human intracellular adhesion molecule ICAM-1 (Horley et al, 1989). ICAM-1 is expressed by lung dendritic cells and seems to be involved in the co-stimulation of $\mathrm{T}$ cells leading to their proliferation (Masten et al, 1997). It has also been shown that dendritic cells were able to migrate from nonlymphoid organs, where they have been stimulated by antigens, to the spleen. This migration is dependent upon the CD11 adhesion molecule (McCarthy et al, 1997). In our experiments, DCs obtained from monocytes cultured with GM-CSF and IL-4 expressed significant levels of CD11c. It is known that CD11c is highly expressed by lung DCs (Nicod and el Habre, 1992). TNF $\alpha$ has been shown to be involved in DC migration by recruiting these cells in the airway epithelia in a model of respiratory infection (McWilliam et al, 1994). Because CD11c can be up-regulated after contact with Der $\mathrm{p} 1$ (the major allergen of Dpt), certainly because of the allergen-induced TNF $\alpha$ production, this adhesion molecule might be involved in the migration of DCs in SCID mice from the lungs (where DCs were exposed to the allergen) the spleen or the lymph nodes (where allergen-positive DCs were detected).

In the last part of this work, we showed that in SCID mice reconstituted intraperitoneally with both DCs and PBMC from allergic patients and exposed to Dpt aerosols, the level of human IgE detected was higher compared with control mice reconstituted with PBMC only. Moreover, in these same mice, the presence of DCs did not modify the IgG production. It is well known that dendritic cells can stimulate CD45+ T cells in the presence of antigen. These $T$ cells are involved in the $\lg \mathrm{E}$ production by providing IL-4, necessary to the switch toward $\lg \mathrm{E}$, and the second signal via CD40-CD40L, involved in the interactions between T cells and B cells (Patel et al, 1996). Moreover, depletion of airway DCs with ganciclovir in thymidine kinase-transgenic mice exposed to ovalbumin aerosols led to a significant decrease of the $\mathrm{lgE}$ production. This suggests that $\mathrm{T}$ cell help for $\mathrm{IgE}$ synthesis was deficient (Lambrecht et al, 1998). Because the production of $\mathrm{IgE}$ is dependent on IL-4, our results suggest that $1 \times 10^{6}$ human dendritic cells introduced via the peritoneal route may amplify a Th2 response.

The presence of DCs in the inflammatory cellular lung infiltrate suggests this cell type is playing an eminent role in the allergic reaction. We can hypothesize a role for DCs in the afferent phase of the immune response by presenting antigens captured in lungs to T cells in lymphoid organs. This may then lead to an increase in the IgE production. The SCID mouse model should be very useful to study the early events leading to sensitization. 


\section{Materials and Methods}

\section{Patients}

Blood was collected from donors sensitive or not sensitive to house dust mites. Allergic patients ( $n=$ 15) presented the usual features of house dust mite sensitization. Skin prick tests toward Dpt were positive, and all patients had serum specific IgE antibodies. Total IgE concentrations were greater than 150 $\mathrm{IU} / \mathrm{ml}(150-1600 \mathrm{IU} / \mathrm{ml})$. Healthy donors $(n=10)$ were tested as negative controls (total lgE levels were less than $150 \mathrm{lU} / \mathrm{ml}$, and they had negative skin prick tests toward commonly inhaled allergens).

\section{Peripheral Blood Mononuclear Cell Preparation}

Platelet rich plasma was obtained after centrifugation (120 ×g, 15 minutes) and discarded. Blood cells were then diluted in RPMI 1640 (Life Technologies, Paisley, Scotland) (vol/vol) and layered over a Ficoll gradient (Pharmacia, Uppsala, Sweden). After centrifugation (400 ×g, 30 minutes), PBMCs were harvested and washed.

\section{Dendritic Cell Differentiation Conditions}

PBMCs were layered on a Percoll gradient (Pharmacia). After centrifugation (400 $\times g, 25$ minutes), monocytes were collected and washed twice in RPMI 1640 medium before plating $\left(2 \times 10^{6}\right.$ cells $/ 2 \mathrm{ml}$ per well) into 6-well flat-bottomed culture plates in RPMI 1640 medium supplemented with $1 \%$ antibiotics and $10 \%$ fetal calf serum (Life Technologies). After 1 hour 30 at $37^{\circ} \mathrm{C}$, nonadherent cells were removed, and adherent cells were cultured at $37^{\circ} \mathrm{C}$ in humidified $5 \% \mathrm{CO}_{2}$ in air, in RPMI medium supplemented with GranulocyteMacrophage colony stimulating factor (GM-CSF) (Peprotech, London, United Kingdom) (20 ng/ml), and in IL-4 (Genzyme, R\&D, Oxon, United Kingdom) (200 $\mathrm{IU} / \mathrm{ml}$ ) for 14 days. GM-CSF and IL-4 were added every 3 days.

\section{Flow Cytometry Dendritic Cell Surface Marker Analysis}

After 14 days of culture in the presence of GM-CSF and IL-4, the cells were collected and incubated for 30 minutes on ice with different monoclonal antibodies: FITC-conjugated CD1a (Dako, Trappes, France), CD80 (Becton Dickinson, Mountain View, California), CD83 (Immunotech, Marseille, France), and PEconjugated CD86 (Becton Dickinson) and CD11c (Becton Dickinson), or with an irrelevant monoclonal antibody of the same isotype (Pharmingen). After washing, the cells were fixed in PBS, 1\% paraformaldehyde and analyzed with an FACScalibur (Becton Dickinson).

\section{Induction of CD11c Expression by Der p 1 Allergen}

DCs were obtained as described above and were cultured in vitro for 7 days in the presence of GM-CSF and IL-4. At day 7, $100 \mathrm{ng} / \mathrm{ml}$ Der $\mathrm{p} 1$ (kindly provided by G.A. Stewart, Perth, Australia) were added in the culture medium every 3 days for 6 days. DCs were then collected and incubated with an FITC-conjugated anti-CD11C antibody for 30 minutes at $4^{\circ} \mathrm{C}$. DCs were washed, fixed in PBS-Paraformaldehyde 1\%, and analyzed by flow cytometry.

\section{Human TNFo Measurement}

DCs were cultured for 7 days and were exposed in vitro to $100 \mathrm{ng} / \mathrm{ml}$ Der $\mathrm{p} 1$ or to $100 \mathrm{ng} / \mathrm{ml}$ tetanus toxin (Boehringer Mannheim) for 24, 48, and 72 hours. Supernatants of $1 \times 10^{6} \mathrm{DCs}$ were assayed for TNF $\alpha$ production. The production of TNF $\alpha$ was measured in culture supernatants by TNF $\alpha$ specific ELISA by using Eli-pairs (R\&D Systems). The sensitivity of detection was $10 \mathrm{pg} / \mathrm{ml}$.

\section{Mice}

C.B.-17 SCID mice (5-7 weeks old) were maintained in isolators (La Calhène, Vélizy, Yvelines, France) with sterilized bedding at the Pasteur Institute of Lille. The SCID colony was regularly checked for absence of mouse serum immunoglobulins.

\section{Peripheral Blood Mononuclear Cells Transfer in SCID Mice: PBMC hu-SCID Mice}

SCID mice were reconstituted by intraperitoneal injection of $10 \times 10^{6}$ mononuclear cells from allergic patients $(n=8)$ or healthy donors $(n=5)$. On the same day, they received intraperitoneally 2 IR Dpt. Four days after the cell reconstitution, one group was exposed to daily allergen aerosols (100 index reactivity [IR] units) for 4 successive days. The control group was not exposed to Dpt. One month later, all mice ( $n=$ 24) were sacrificed, and lungs, spleen, and thymus were recovered. The excised organs were fixed in $4 \%$ buffered paraformaldehyde and dehydrated before freezing or inclusion in paraffin.

\section{Dendritic Cell Transfer in SCID Mice: DC hu-SCID Mice}

Dendritic cells $\left(1 \times 10^{6}\right)$ obtained from in vitro culture of monocytes from allergic patients $(n=7)$ or healthy donors $(n=5)$ were transferred intraperitoneally into naive SCID mice. One group of mice was exposed to Dpt by two aerosols performed 4 days after the mice reconstitution. The second group used as a control was not exposed to allergen inhalation.

Seven days after the cell reconstitution, SCID mice ( $n=28$ ) were sacrificed and their lungs, spleen, and thymus were removed. The excised organs were fixed in $4 \%$ buffered paraformaldehyde and dehydrated before freezing or inclusion in paraffin.

\section{DCs and PBMC Transfer in SCID Mice: DCs+PBMC hu-SCID Mice}

Naive SCID mice were reconstituted at Day 0 with $1 \times$ $10^{6}$ human dendritic cells obtained from monocytes in culture. A group of mice not reconstituted with human DCs was used as a negative control. At Day 5, PBMC of the same donors (allergic asthmatics $[n=4]$ or 
healthy donors $[n=4])$ and 2 IR Dpt were injected in the peritoneal cavity of all mice. At Day 9, all mice were exposed to a daily aerosol of Dpt for 4 days. Blood was collected from all mice each week for 4 weeks.

\section{Immunohistochemical Analysis of the Organs}

Serial frozen or paraffin tissue sections (6 $\mathrm{mm}$ thick) were performed on the different collected organs. After a 20 minutes permeabilization in Tris buffered saline (TBS)-Triton $0.3 \%$, the slides were saturated for 1 hour at room temperature with TBS-20\% normal human serum. Sections were incubated for 1 hour with the following primary antibodies specific for human markers: CD1a (Dako), CD83 (Immunotech), HLAclass II (Dako), CD68 (Dako), CD45 (Becton Dickinson), DC-LAMP (specific for mature DCs), and DCGM4 (specific for immature DCs, kindly provided by Serge Lebecque; Schering Plough, Dardilly, France) or an isotype-matched mouse IgG (Dako) as a negative control. After saturation with $10 \%$ normal human serum diluted in TBS, sections were stained using an APAAP technique (Dako) previously described (REF) (Duez et al, 1996), repeated once to increase the sensitivity of the reaction. All slides were lightly counterstained with hematoxylin (Sigma, St Louis, Missouri). Cells were counted in at least two different grids at a magnification of $\times 250$, corresponding to $1 \mathrm{~mm}^{2}$. The number of human cells was expressed as the mean \pm SEM per $\mathrm{mm}^{2}$. The antibodies used in this study did not cross react with murine tissues.

\section{Detection of Allergen-Pulsed DCs}

Serum was collected from rabbits immunized with a peptide (ie, peptide 117-133) of Der p 1 (Jeannin et al, 1992), a major allergen of the house dust mite Dpt, and was tested in immunohistochemistry for the detection of allergen-positive DCs. Because CFA was used as adjuvant in the peptide immunization protocol, serum from rabbits immunized with a nonimmunogenic peptide of Der p 1 (peptide 176-187) was used as potential negative control to exclude a nonspecific immune reactivity. Using Western blot this negative control serum failed to recognize Der $p$ 1. Sections of spleen and peripheral lymph nodes (inguinal lymph nodes) were permeabilized for 20 minutes with TBSTriton $0.3 \%$. After inactivation of endogen peroxidases with TBS $-\mathrm{H}_{2} \mathrm{O}_{2} 1 \%$, the slides were incubated for 1 hour with 1/100 of the rabbit serum diluted in TBS at room temperature. They were then incubated with a peroxidase-conjugated secondary antibody (Sigma) for 30 minutes. Sections were stained using the peroxidase substrate DAB (Vector Laboratories, Burlingame, California). All slides were lightly counterstained with hematoxylin.

\section{IgE/IgG Measurement in DCS + PBMC hu-SCID Mice}

The detection of total human IgE was investigated by using a CAP-system method (Pharmacia). The sensitivity of detection was $0.1 \mathrm{IU} / \mathrm{ml}$. The detection of total human IgG was investigated by nephelometry. The sensitivity of the method allows the detection of low levels of $\operatorname{lgG}(1 \mathrm{mg} / \mathrm{ml})$.

\section{Statistical Analysis}

Results are expressed as the mean \pm SEM. Parametric statistical analysis of dendritic cells in lungs, spleen, and thymus of mice exposed or not exposed to Dpt aerosols and analysis of the $\mathrm{lgE} / \mathrm{lgG}$ production were performed using the Wilcoxon test. Values of $p \leq 0.05$ were considered as statistically significant.

\section{Acknowledgements}

The authors wish to thank the personnel of the Colmelte Hospital for the selection of patients and the blood collection involved in this study, Philippe Marquillies for assistance in animal experiments and Sophie Galand for help in typing and collecting references. The authors are grateful to Philippe Gosset and Adrieu Tomkinson for critical review of this work.

\section{References}

Abbas AK, Murphy KM, and Sher A (1996). Functional diversity of helper T lymphocytes. Nature 383:787-793.

Ban M, Hettich D, Goutel M, and Bonnet P (1997). TDI inhalation in guinea-pigs involves migration of dendritic cells. Toxicol Letters 93:185-194.

Barratt-Boyes SM, Watkins SC, and Finn OJ (1997). In vivo migration of dendritic cells differentiated in vitro. A chimpanzee model. J Immunol 158:4543-4547.

Bellini A, Vittori E, Marini M, Ackerman V, and Mattoli S (1993). Intraepithelial dendritic cells and selective activation of Th2-like lymphocytes in patients with atopic asthma. Chest 103:997-1005.

Cella M, Sallusto F, and Lanzavecchia A (1997). Origin, maturation and antigen presenting functions of dendritic cells. Curr Opin Immunol 9:10-16.

Duez C, Akoum H, Marquillies P, Tonnel AB, and Pestel J (1998). Allergen-induced migration of human cells in allergic SCID mice. J Scand Immunol 47:110-115.

Duez C, Kips J, Pestel J, Tournoy K, Tonnel AB, and Pauwels $R$ (2000). House dust mite induced airway changes in HuSCID mice. Am J Respir Crit Care Med 161:200-206.

Duez C, Tsicopoulos A, Janin A, Tillie-Leblond I, Thyphronitis G, Marquillies P, Hamid Q, Wallaert B, Tonnel AB, and Pestel $J(1996)$. An in vivo model of allergic inflammation: Pulmonary human cell infiltrate in allergen-challenged allergic Hu-SCID mice. Eur J Immunol 26:1088-1093.

Goodman DJ and Gerondakis S (1997). Simultaneous expression of germline gamma and epsilon immunoglobulin heavy chain transcripts in single murine splenic B cells. Mol Immunol 34:919-927.

Horley KJ, Carpenito C, Baker B, and Takei F (1989). Molecular cloning of murine intracellular adhesion molecule (ICAM-1). EMBO J 8:2889-2896. 
Jeannin P, Delneste $Y$, Buisine E, Le Mao J, Didierlaurent A, Stewart GA, Tartar A, Tonnel AB, and Pestel J (1992). Immunogenicity and antigenicity of synthetic peptides derived from the mite allergen Der $\mathrm{p} I$. Mol Immunol 30:15111518.

Kalinski P, Schuitemaker JH, Hilkens CM, and Kapsenberg ML (1998). Prostaglandin E2 induces the final maturation of IL-12-deficient CD1a+CD83+ dendritic cells: The levels of $\mathrm{IL}-12$ are determined during the final dendritic cell maturation and are resistant to further modulation. J Immunol 161:28042809.

Lambrecht BN, Salomon B, Klatzmann D, and Pauwels RA (1998). Dendritic cells are required for the development of chronic eosinophilic airway inflammation in response to inhaled antigen in sensitized mice. J Immunol 160:4090-4097.

Leenen PJM, Radosevic K, Voerman JSA, Salomon B, van Rooijen N, Klatzmann D, and van Ewijk W (1998). Heterogeneity of mouse spleen dendritic cells: In vivo phagocytic activity, expression of macrophage markers and subpopulation turnover. J Immunol 160:2166-2173.

Lore K, Sonnerborg A, Spetz AL, Andersson U, and Andersson J (1998). Immunocytochemical detection of cytokines and chemokines in Langerhans cells and in vitro derived dendritic cells. J Immunol Methods 214:97-111.

Masten BJ, Yates JL, Pollard Koga AM, and Lipscomb MF (1997). Characterization of accessory molecules in murine lung dendritic cell function: Roles for CD80, CD86, CD54, and CD40L. Am J Respir Cell Mol Biol 16:335-342.

McCarthy DA, Macey MG, Bedford PA, Knight SC, Dumonde DC, and Brown KA (1997). Adhesion molecules are upregulated on dendritic cells isolated from human blood. Immunology 92:244-251.

McWilliam AS, Nelson D, Thomas JA, and Holt PG (1994). Rapid dendritic cell recruitment is a hallmark of the acute inflammatory response at mucosal surfaces. J Exp Med 179:1331-1336.
Moller GM, Overbeek SE, Van Helden-Meeuwsen CG, Van Haarst JM, Prens EP, Mulder PG, Postma DS, and Hoogsteden HC (1996). Increased numbers of dendritic cells in the bronchial mucosa of atopic asthmatic patients: downregulation by inhaled corticosteroids. Clin Exp Allergy 26:517-524.

Nicod LP and el Habre F (1992). Adhesion molecules on human lung dendritic cells and their role for T-cell activation. Am J Respir Cell Mol Biol 7:207-213.

Palucka KA, Taquet N, Sanchez-Chapuis F, and Gluckman JC (1998). Dendritic cells as the terminal stage of monocyte differentiation. J Immunol 160:4587-4595.

Patel HR, Oshiba A, Jeppson JD, and Gelfand EW (1996). Differential expression of CD40 ligand on $T$ cell subsets. Implications for different roles of CD45RA+ and CD45RO+ cells in IgE production. J Immunol 156:1781-1787.

Pestel J, Jeannin P, Delneste Y, Dessaint JP, Cesbron JY, Capron A, Tsicopoulos A, and Tonnel AB (1994). Human IgE in SCID mice reconstituted with peripheral blood mononuclear cells from Dermatophagoides pteronyssinus-sensitive patients. J Immunol 153:3804-3810.

Semper AE, Hartley JA, Tunon-de-Lara JM, Bradding P, Redington AE, Church MK, and Holgate ST (1995). Expression of the high affinity receptor for immunoglobulin $E$ (lgE) by dendritic cells in normals and asthmatics. Adv Exp Med Biol 378:135-138.

Van Haarst JM, de Wit HJ, Drexhage HA, and Hoogsteden HC (1994). Distribution and immunophenotype of mononuclear phagocytes and dendritic cells in the human lung. Am J Respir Cell Mol Biol 10:487-492. 